\title{
Spectral manifestation of distorted forms metalloporphyrins at low temperatures
}

\author{
Aleksander Starukhin ${ }^{1,}$, , Aleksander Gorski ${ }^{2}$, and Michal $\mathrm{Kijak}^{2}$ \\ ${ }^{1}$ B.I. Stepanov Institute of Physics, National Academy of Sciences of Belarus, Minsk, Belarus \\ ${ }^{2}$ Institute of Physical Chemistry, Polish Academy of Sciences, Warsaw, Poland
}

\begin{abstract}
Different spectral forms of a set of metalloporphyrins with $\mathrm{Mg}$ (II), Zn (II), Pd (II) and Pt (II) ions in solid solutions (Shpol'skii matrices, solid tetrahydrofuran and solid rare gas matrices) have been detected in the absorption and luminescence spectra at cryogenic temperatures. The spectral manifestation of a planar form, as well as two distorted forms for $\mathrm{Mg}$ - and Zn-porpyrins was formed in the ground electronic state at low temperatures. An existence of two spectral forms of Pd and Pt-porphyrins was observed in their phosphorescence spectra. In the case of Mg- porphyrin the spectral shift between positions of $0-0$ transitions the planar and first distorted spectral forms is about $220 \mathrm{~cm}^{-1}$.
\end{abstract}

Metallocompexes of porphyrins are biologically relevant compounds known for many years. It is well known, that metallocomplexes of porphyrins demonstrate planar structures of high symmetry at room temperature in neutral solvents. However, under certain conditions, the planar structure of the porphyrin macrocycle may be distorted, that leads to corresponding spectral manifestations of the nonplanarity effects. First, the method of resonance Raman spectroscopy was used for recording and analysing of distorted structures of metalloporphyrins [1]. Nevertheless, the mentioned method does not have selectivity for analysing of the manifestation of several types of distorted forms together. In contrast, the methods of luminescent spectroscopy may be effectively used in a cases of stabilization of various (including liganded) spectral forms at low temperatures. Fluorescence line narrowing procedure at low temperatures [3] permits excites selectively and registers the highly resolved spectra of individual conformers and assigns the frequencies of normal modes.

In this report we were discussing progress in study of metallocomplexes of porphine (P), octamethylporphyrin and octaethylporphyrin in different solid matrices at cryogenic temperatures. Spectral manifestation of different forms have been investigated and characterized by methods of luminescent and fine line spectroscopy.

For example, three spectral forms with positions of $0-0$ transition located: at 552,4 nm (form F0), 562,4 (form FI) and 566,5 (form FII) have been detected in fluorescence spectra of Zn-porphyrin (Zn-P) in solid Ar at $4.2 \mathrm{~K}$. A spectral manifestation of above mentioned forms is due to are stabilized of planar (form F0) and two different distorted FI and FII conformations of $\mathrm{Zn}-\mathrm{P}$ in low temperatures matrices. Forming of these forms in the ground state has been proved early for $\mathrm{Mg}-\mathrm{P}$ and $\mathrm{Zn}-\mathrm{P}$ upon selective detection of fluorescence

\footnotetext{
Corresponding author: a.starukhin@ifanbel.bas-net.by
} 
excitation and fluorescence spectra (3). For Zn-P distorted forms have been detected in the fluorescence and phosphorescence spectra simultaneously. The manifestation of the two forms spectra of the Pd- and Pt-P has been observed in the phosphorescence and excitation of fluorescence and phosphorescence spectra [4] with spectral shift of the $0-0$ positions about $78 \mathrm{~cm}^{-1}$ and $38 \mathrm{~cm}^{-1}$, respectively.

One of the most fascinating results is the detection of absorption (fluorescence excitation) fine line spectra of Mg-P and Zn-P of Soret band of (Fig. 1).
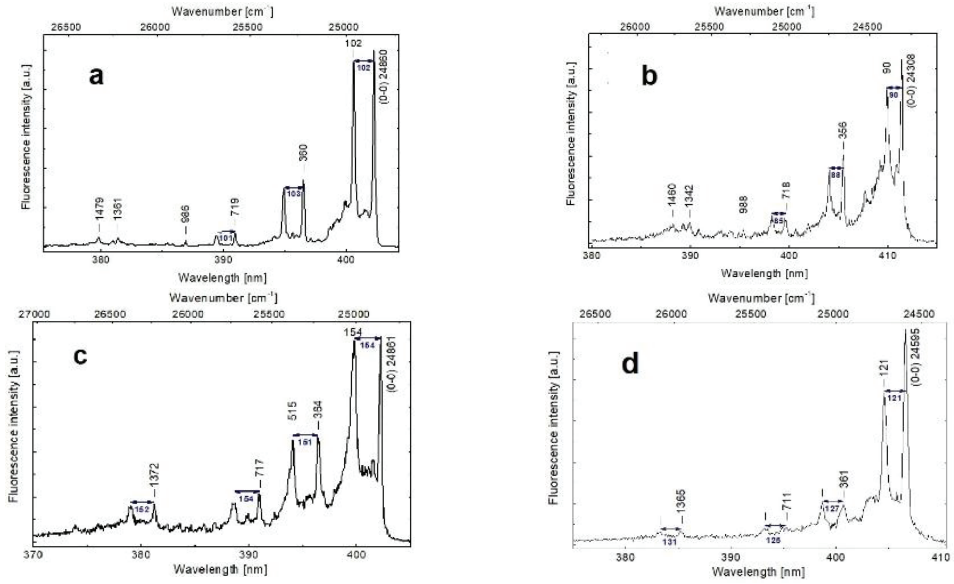

Fig. 1. Fluorescence excitation spectra of Mg-P and $\mathrm{Zn}-\mathrm{P}$ in the range of Soret band of Form I (a - Mg$\mathrm{P}, \mathbf{c}-\mathrm{Zn}-\mathrm{P}$ ) and Form II (b - Mg-P, d - Zn-P in solid matrix of tetrahydrofurane (THF) at 4,2 K

The set of vibrational modes of the excited state (Soret band) was detected, e.g. for FI form of Mg-P vibronic lines with frequencies 360, 719, 986, 1361 and $1479 \mathrm{~cm}^{-1}$ (Fig. 1a). Interestinglly, that vibronic bands are split into doublets with a constant separation, specific for a given form and type of a central ion. Density Functional Theory (DFT) and time dependent DFT methods were used for moddeling of the ground and electronically excited states of investigated mettalocomplexes. Virbational analysis was performed to explaine observed spectral data. Based on that, an assignment of experimentally observed forms was proposed. The FI form was attributed to a complex of metalloporphyrin with one extra ligand placed above its plane. The FII form corresponds to a structure with two additional ligands located on opossite sides of the porphyrin macrocycle. Jahn-Teller distortions of studied molecules in Q and Soret states and resulting band splitting has been also analysed.

This work was supported by the BRFFI Grant No. F16R-084 and Horizon 2020 MSCA RISE Grant 645628 .

\section{References}

[1] J.A. Shelnutt et al., Chem. Soc. Rev. 27, 31 (1998)

[2] R.I. Personov, E.I. Al'shits, L.A. Bykovskaya, Opt. Commun. 7, 169 (1973)

[3] A.S. Starukhin, A.M. Shul'ga, Opt. and Spectrosc. 98, 780 (2005)

[4] A. Starukhin, M. Kruk, R. Czerwieniec, J. Lumin. 128, 531 (2008) 